Micron and Microscopica Acta, vol. 16, No. 3, pp. 193-194, 1985. Printed in Great Britain.
$0739-6260 / 85 \$ 3.00+0.00$

(C) 1985 Pergamon Press Ltd.

\title{
ELECTRON MICROSCOPY FOR EXFOLIATIVE CYTOLOGY AND FINE NEEDLE ASPIRATION
}

\author{
Ted F. Beals, M.D.
}

Clinical Electron Microscopy Unit, Veterans Administration Medical Center

Department of Pathology, Medical School The University of Michigan, Ann Arbor

The cytopathologist examines a variety of specimens from patients suspected of having neoplasms, as well as large numbers of individuals who have a high risk for developing malignancy. The routine PAP smear (cervical-vaginal scrapping) and sputum smears are the most common of these specimens. Over the years numerous cytopathologists and some electron microscopists have adapted procedures which allow ultrastructural examination of the full spectrum of cytology preparations. In the Clinical Electron Microscopy Unit at the Ann Arbor Veterans Administration Medical Center we have processed more than 800 such specimens, predominantly non-gynecological and with a high frequency of malignancy. In this brief overview of the subject I will point out some of the situations in which ultrastructural examination has proven, over time, to be of greatest benefit to cytodiagnosis.

Traditionally cytology specimens are subdivided into: exfoliative (those which contain superficial cells shed from exposed mucosa), fluids (generally these are normally occurring fluids which may contain a variety of living cells and are a frequent site for metastatic neoplasms), and the more recently introduced fine needle aspirations (in which cells are mechanically pulled from solid tissues for examination in smears). In our own and other's experience, the later two types of specimens are best suited for examination in the transmission and scanning electron microscopes (TEM,SEM). Of our 809 cytology specimens examined by electron microscopy, $261(32 \%)$ were fine needle aspirates, while $174(22 \%)$ were serous fluids. In our medical center fine needle aspirates comprise $2.5 \%$ of the cytology specimens and serous fluids $5.5 \%$. The most significant reason for the predominance of electron microscopic examinations of these two types of specimens are: they contain a greater number of viable cells (exfoliative cells are frequently degenerated or dead), and the frequency of malignancy is much greater.

Sputa, urine, cerebrospinal fluid and brushings and washings from the respiratory and gastrointestinal tracts can all be processed for examination in the TEM, SEM or both. Although viruses, microorganisms and assorted intra- and extracellular objects can be studied in the TEM the principle role for electron microscopy is in cell identification and specifically in the subclassification of neoplasms. For details of techniques and a more comprehensive discussion, see the reviews of Beals (1983) and Ordonez (1981).

Serous fluids are easily processed for both SEM and TEM. There are large numbers of free floating cells and the diagnostically significant cells are usually abundant. Filters collect the celis readily and facilitate the examination in both SEM and TEM. However, centrifuged cell pellets are more productive for TEM because of the increase in sample volume and cellular density. We have found the electron microscope is most beneficial in distinquishing metastatic adenocarcinomas from reactive mesothelial cells (this is a significant light microscopic problem in a few specimens), and in distinquishing adenocarcinomas from mesotheliomas. Many reports suggest that the ultrastructural characteristics of cases of adenocarcinoma of unknown primary are useful in identifying the site of origin. Our experience with more than 80 malignant effusions suggest that although such features may suggest a possible site of origin, they can not be used as definitive diagnoses. 0ccassional specific cell types, such as melanoma or alveolar cell carcinoma, can be specifically distinquished in the TEM when the cell type is uncertain in the light microscope. Such situations are, however, extremely rare in clinical practice. 
SEM of fine needle aspirates has proven to be of little value (probably because cell surfaces are usually badly damaged during the aspiration process). TEM, on the other hand has been very useful as an aid to identification of neoplasms, and cells of ambiguous type seen in the light microscopic preparations. In 88 aspirates (22\% of the 229 examined by TEM) the ultrastructural characteristics improved the diagnosis made by cytology alone, and in 23 aspirates ( $6 \%$ of the total) the diagnosis was changed after examination in the TEM.

Careful correlation of the light and electron microscopic features of cytology specimens is necessary to realize the full value of the characteristics seen, as well as to reduce the incidence of misinterpretations (and potential misdiagnosis). Although a strong argument can be made that meaningful ultrastructural analysis is only possible when the specific cell or cells under consideration in the light microscope are studied in the electron microscope, this is impractical in clinical practice. Alcoholic fixation is a prerequisite for accurate cytodiagnosis. With rare exceptions (such as virus identification) this fixative is unacceptable for ultrastructural analysis. Unfortunately, a universal cytology fixative (as is now routinely used in surgical pathology) has not been developed.

Since such a small percentage of cytology specimens will benefit from electron microscopic examination, it is prohibitively expensive to parallel process all of our patient specimens. This means selection of cases for additional processing; and meaningful selection is only possible after the specimen has been examined in the light microscope and determined to be a "problem case". With fine needle aspirations the percentage of cases which will benefit significantly from electron microscopy is much greater (in our experience, 24\%). Therefore, routinely processing of all aspirates is justified. We accomplish this by fixing the rinsings from the needle and syringe used in the aspiration in glutaraldehyde and by centrifugation forming cell blocks which are then processed into plastic. All of these blocks are cut with one micron thick (semithin, toluidine blue stained) sections for light microscopic findings. The enhanced light microscopic images possible with plastic embedding are reason enough for using this procedure; the possibility for TEM examination is a fringe benefit.

Nearly all cytology laboratories routinely save the unused aliquote from their specimens, under refrigeration, for at least 24 hours. This provides a practical source for further analysis if questions remain following examination of the Papanicolaou stained smears. We have found little change of diagnostic significance in such specimens, even 72 hours after collection, when subsequently processed for electron microscopy.

Although the decreased availability of SEMs is a handicap, we have found that distinquishing atypical mesothelial cells from adenocarcinomas is frequently possible with SEM alone. Processing is possible in less than six hours and the technical expenses are minimal since embedding and sectioning are not necessary. In the SEM it is also possible to survey hundreds of cells rapidly, thereby facilitating interpretations. Nearly all other cell identifications are more accurately performed in the TEM.

Unfortunately, electron microscopy has not been found to be clinically useful in distinquishing malignant from benign cells. Its greatest contribution has been to further our understanding of the cellular characteristics seen in the light microscope and in increasing our level of confidence in the cytodiagnosis of cell types. Occassional cases can be significantly aided by electron microscopy. Our experience in a hospital-based, predominantly non-gynecological cytology laboratory suggests that about $2 \%$ of cytology diagnoses will be influenced by selective ultrastructural examination of specimens. Less than $1 \%$ of cytodiagnoses will be significantly changed by such examination. But, unless electron microscopy laboratories take the time to learn the special techniques needed to process the various types of cytology specimens and are willing to expidite the processing and examination of patient specimens, the value of electron microscopy for cytodiagnosis will be lost.

\section{REFERENCES}

Beals, T.F. (1983). Cytology and electron micrscopy. In B.F.Trump and R.T.Jones (Eds), Diagnostic Electron Microscopy, Vo 1.4. John Wiley and Sons, New York, pp 179-225. Ordonez, N.G. (1981). Electron microscopy in cytodiagnosis. In Introduction to Diagnostic Electron Microscopy. B. Mackay. Appleton-Century-Crofts, New York, pp211-219. 